\title{
Synergistic Effect of ELF-Magnetic Field and IR-Radiation on Tinnitus Patients
}

\author{
D. Hojan-Jezierska ${ }^{a, b, *}$, M. Pankowska ${ }^{a}$, A. Wicher $^{c}$, E. SzymieC $^{d}$, M. Kraśnik $^{a}$ \\ AND M. KNAPKIEWICZ ${ }^{a}$ \\ ${ }^{a}$ Department of Biophysics, Poznań University of Medical Sciences, Poznań, Poland \\ ${ }^{b}$ Higher Vocational State School, Piła, Poland \\ ${ }^{c}$ Institute of Acoustics, A. Mickiewicz University, Poznań, Poland \\ ${ }^{d}$ ENT Department, Poznań University of Medical Sciences, Poznań, Poland
}

\begin{abstract}
The LED light therapy and magnetostimulation is an innovative method of treatment used in Viofor JPS System. Simultaneous application of both types of electromagnetic radiation increases the therapy effectiveness by the synergy effect. It is recommended in analgesic, anti-inflammatory treatment, and to support immune system of the organism. The mechanism of biological effect of variable magnetic fields and light are of similar nature at the tissue level. When applying magnetostimulation and light together, it shortens the time of therapy. The LED light therapy and magnetostimulation is supposed to be an alternative supporting method to therapies used to treat tinnitus so far.
\end{abstract}

PACS: 87.50.C-, 87.50.ct

\section{Introduction}

In the manuscript, an attempt of usefulness estimation of Viofor JPS System Magnetic \& Light Therapy for tinnitus patients was made. Patients were treated with the light and magnetic field via the infrared magnetic-light applicator IR. The wavelength of light was $855 \mathrm{~nm}$.

Patients with lateralized tinnitus participated in the experiment. The distortion product otoacoustic emission method (DPOAE) was the objective measure of influence of LED light therapy and magnetostimulation on the auditory system condition. For the first time authors used also the visual analog scale for these estimations.

\subsection{Extremely low frequency (ELF)-magnetic field and IR-radiation therapy}

ELF magnetotherapy has been introduced as complementary treatment to other methods of physical therapy. It is also one of the basic methods of medical rehabilitation.

Light energy has mainly a topical effect on tissues. Its penetration inside the body depends on the light wavelength. Tissue reaction depends on energy absorption in each tissue layer. The absorption effectiveness is mainly affected by: tissue thickness, blood supply and flow, water content and presence of pigment. Infrared reactions start at the level of cell membrane.

Nowadays the magnetostimulation with energy of light is the object of increasing interest. There are investigations whose results prove the positive effect of this kind of therapy [1-4].

* corresponding author; e-mail: djeziers@ump.pl
The biological effects of varying magnetic field result from electrodynamic effect on ion currents in the body, magnetomechanic effect and ionic cyclotron resonance of ions in the body fluids. At the tissue level, mechanism of biological effect of variable magnetic fields and light energy are of similar nature. When applying magnetostimulation together with light, a synergetic effect could be expected, which can be particularly useful in tinnitus rehabilitation. The wider spectrum of magnetic field can support a strong, local effect of light.

Main biological effects of magnetostimulation paired with light energy should be among others: the improved oxygen assimilation by its carriers, which improves adenosine-5'-triphosphate (ATP) synthesis in oxidation-reduction systems of aerobic and anaerobic respiration path; strong stimulation of DNA synthesis and cell proliferation. In case of tinnitus patients very important seems to be restored ATP-dependent membrane pump activity, which has been lost due to disease-related changes. For sodium-potassium and calcium pumps in neurons, it leads to membrane hyperpolarization and stimulus blocking, even those of high amplitude; as a result, pain stimuli conduction in afferent fibers is decreased, therefore a clear analgesic effect can be observed.

Changed activity of serotoninergic synapses and stimulation of beta-endorphin release, and stimulation of pituitary gland-adrenal gland axis accompanied by increased release of cyclocorticoids adds to a strong analgesic effect.

Magnetostimulation with energy of light directly effects on liquid crystal membrane structure and on modification of membrane enzyme activity related with increased ATP-dependent ion pumps through increased ATP synthesis in mitochondria. Each of the above pro- 
cesses leads to changed membrane permeability, thus to change in electrolyte and water distribution between cells or their organelle and surrounding areas.

One of the newest devices used for magnetostimulation with energy of light is the Viofor JPS Magnetic \& Light Therapy (Clinic Delux). It was used in investigations.

\subsection{Tinnitus}

Tinnitus is a conscious experience of sound that originates in the head, without any corresponding external source. Tinnitus noises are described as ringing, whistling, buzzing or humming. The noise may be continuous or it may come and go. There may be a single noise or two or more components. The noise may be heard in one ear, both ears or at the centre of the head.

All theories trying to explain the genesis of tinnitus, converge to the existence of an aberrant excitatory activity in the auditory pathways. It is obvious that more than one complex mechanism may be involved in the generation and perception of the symptom. The understanding of these mechanisms is crucial to therapeutic success [5].

Many complementary and alternative therapies have been proposed. It is worth mentioning acupuncture, aromatherapy, homeopathy, or herbal medicine. Also, behavioural techniques are used to tame tinnitus. The tinnitus suppressive effect of electric stimulation and repetitive transcranial magnetic stimulation has been observed in some patients $[6,7]$. But there is still no effective therapeutic method for tinnitus.

\subsection{Otoacoustic emission}

Objective evaluation of the hearing system can be performed with the use of tonal audiometry, auditory brainstem response, or acoustic otoemissions. Particularly promising is the method based on the DPOAE method as it is related to the phenomenon of tinnitus $[8,9]$.

The DPOAE is believed to arise from the nonlinear work of the cochlea stimulated by signals with frequencies $f_{1}$ and $f_{2}$. The generation of DPOAE is connected with the processes taking place in the cochlea responsible for the amplification of vibrations on the basilar membrane. Due to intermodulation, the cochlea generates a long series of components which are not present in the input stimuli. These components are called distortion products.

In the present study, attempts are made to determine the impact of magnetostimulation paired with energy of light on the level of DPOAE in patients with tinnitus.

\subsection{Visual analog scale}

A visual analog scale (VAS) is a measurement instrument that tries to measure a characteristic or attitude that is believed to range across a continuum of values and cannot easily be directly measured. For example, the amount of pain that a patient feels ranges across a continuum from none to an extreme amount of pain. From the patient's perspective this spectrum appears continuous. Their pain does not take discrete jumps, as a categorization of none, mild, moderate and severe would suggest.
It was to capture this idea of an underlying continuum that the VAS was devised [10-12].

Operationally, a VAS is usually a horizontal line, $100 \mathrm{~mm}$ in length, anchored by word descriptors at each end. The patients mark on the line the point that they feel represents their perception of their current state.

The score from 0 to 10 on the VAS was consider to represent an equivalent distance on the VAS in centimetres. We chose $1.0 \mathrm{~cm}$ as the limits of agreements.

In the present study the VAS was used for estimation of influence of magnetostimulation with energy of light on tinnitus patients. Visual analog scale is presented in Fig. 1.

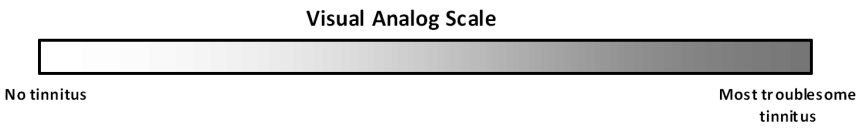

Fig. 1. Visual analog scale.

\section{Experimental procedure}

The study on magnetostimulation paired with energy of light on the human body was approved by the local Ethical Committee.

The group of 6 tinnitus patients (tinnitus) participated in the studies of magnetic field paired with light energy influence on auditory system. The age range was within 17-75 years. Tinnitus patients were selected from those treated at the Centre of Laryngological Rehabilitation Medical University in Poznań. All participants expressed written formal consent to take part in the study. In order to estimate their wellness and possible contraindications to participate in the experiment, the subjects were examined by means of a specific questionnaire. Prior to magnetostimulation patients were examined using a tonal audiometer. Next, the tinnitus pitch and tinnitus loudness were measured. In our experiment the tinnitus matching stimuli were presented to the ipsilateral ear using the same audiometer as in the hearing threshold measurements, with the subject positioned in a sound-proof booth. Depending on the sensation of the tinnitus pitch, pure tones or narrow band-pass noises were used as matching stimuli. They were presented at level corresponding to the tinnitus loudness. All data for patients are collected in Table.

Furthermore, the DPOAE audiograms (DP-grams) were recorded in soundproof room by Audera GrasonStadler system. Primary tones were presented at the level of $L_{1}=65 \mathrm{~dB}$ SPL and $L_{2}=55 \mathrm{~dB}$ SPL. The $f_{2} / f_{1}$ ratio was set on 1.22 . The responses were measured at $2 f_{2}-f_{1}$ with respect to $f_{2}$. The DP-grams were collected in the $f_{2}$ frequency range from 1 to $8 \mathrm{kHz}$ and they were accepted as valid when the signal-to-noise ratios of the $2 f_{1}-f_{2}$ component exceeded $6 \mathrm{~dB}$. 
Subject characteristics.

TABLE

\begin{tabular}{c|c|c|c|c|c|c}
\hline \hline $\begin{array}{c}\text { Patient } \\
\text { no. }\end{array}$ & $\begin{array}{c}\text { Age } \\
\text { [years] }\end{array}$ & $\begin{array}{c}\text { Frequency } \\
\text { of noise } \\
{[\mathrm{Hz}]}\end{array}$ & $\begin{array}{c}\text { Level } \\
\text { of noise } \\
{[\mathrm{dB}]}\end{array}$ & $\begin{array}{c}\text { Kind } \\
\text { of noise }\end{array}$ & Lateralization & $\begin{array}{c}\text { Hearing } \\
\text { loss }\end{array}$ \\
\hline 1. & 57 & 6000 & 83 & tonal & right ear & yes \\
2. & 21 & 6000 & 75 & tonal & right ear & yes/no \\
3. & 62 & 8000 & 78 & tonal & right ear & yes \\
4. & 64 & 4000 & 60 & tonal & right ear & yes \\
5. & 17 & 8000 & 10 & tonal & right ear & no \\
6. & 75 & 250 & 34 & narrow-band & left ear & yes
\end{tabular}

The intensity level of primary tones was held constant at all frequencies tested and DPOAE data were plotted for different frequencies. Furthermore, patients marked on the VAS the point that they feel represents their perception of the current state for tinnitus.

Next they were treated by Viofor JPS which offers magnetic field paired with light energy, in the program M2P3 (Fig. 2), using the infrared magnetic-light applicator IR, where the $M$ stands for application mode and $\mathrm{P}$ for the program of the Viofor JPS.

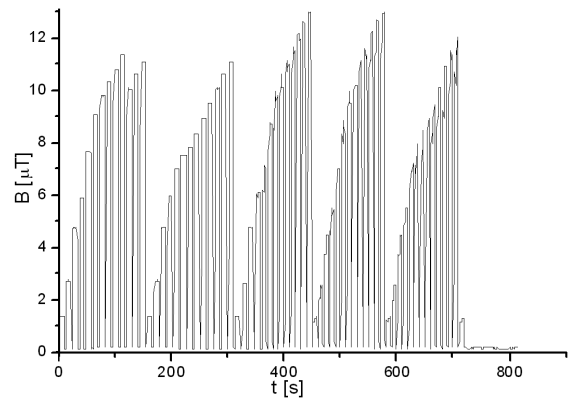

Fig. 2. Changes in the magnetic field ( $z$-component) for the magnetic field course in the M2P3.

After the magnetostimulation, the patients' DP-grams were measured again to determine changes in DPOAE's amplitudes and patients marked they feelings on the VAS again.

\section{Results and discussion}

For the patients participating in experiment the tinnitus appeared as a tonlike sensation at the frequency range $4-8 \mathrm{kHz}$ in 5 cases and in one case as a noise-like sensation with a central frequency of a noise band $250 \mathrm{~Hz}$. The DPOAE levels, before and after the procedure of magnetostimulation paired with light energy, were compared. The 3 factor variance analysis showed that the factor "magnetostimulation" was statistically insignificant $(p=0.564)$. This fact can be simply explained taking into consideration the hearing loss of patients. In most sensory hearing losses one can observe the absence of otoacoustic emissions. In the previous research besides the DPOAE subjects were examined by means of a specific questionnaire [9]. The analysis of questionnaires allowed to describe changes in severity of tinnitus only in words. Authors did not use other measure to determine changes in patients state.

In the present research to evaluate the influence of magnetostimulation the VAS also was analysed. This method of rating is thought to provide greater sensitivity for measurements of subjective phenomena [10-12]. The values indicated by patients on the scale before and after the magnetostimulation paired with light energy are shown in Fig. 3.

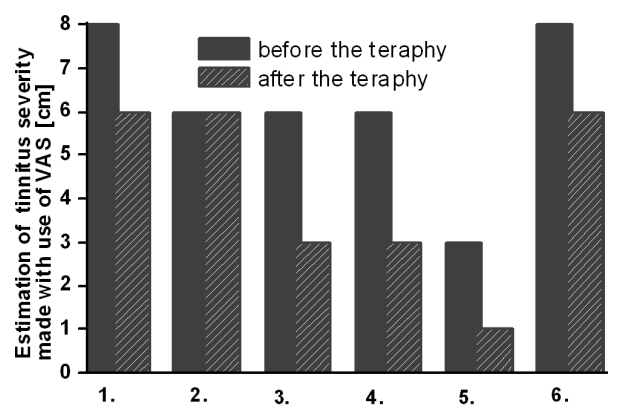

Fig. 3. Estimation of tinnitus severity made by patients with the use of VAS.

The statistical analysis of results gained using the VAS method was performed. The Wilcoxon signed ranks test indicated that the levels of severity of tinnitus were lower after than before the therapy $(p=0.03125)$.

\section{Conclusions}

On the basis of the above presented results we are able to draw the following conclusions:

1. No statistically significant influence of ELF magnetostimulation with light energy on the level of DPOAE amplitudes has been observed. It is very hard to measure otoemission for patients with hearing loss. 
2. The use of VAS for tinnitus patients seems to be good step for evaluation of such a subjective sensation.

3. It is considerable to divide patients for different groups taking into account the lateralization of tinnitus.

4. The preliminary results show the possibility of use of VAS as the complementary method for the questionnaire, especially in the case of patients with hearing losses. The VAS has been used as an effective method to quantify the condition of the subject. Ultimate success in treating patients with magnetostimulation should include each patient's perception of the severity of tinnitus, highlighting the significance of patient-assessment data such as VAS.

\section{References}

[1] A. Sieroń, $O P M$ 5, 55 (2005).

[2] A. Sieroń, J. Pasek, R. Mucha, Rehabilitacja w Praktyce 3, 20 (2006) (in Polish).
[3] J. Pasek, R. Mucha, A. Sieroń, Acta Bio-Optica Inform. Med. 1, 15 (2006).

[4] J. Pasek, R. Mucha, A. Sieroń, Acta Bio-Optica Inform. Med. 12, 93 (2006).

[5] O.C. Person, M.C.C. Féres, Neurobiologia 68, 4 (2005).

[6] B. Langguth, G. Hajak, T. Kelinjung, S. Pridmore, P. Sand, P. Eichhammer, Acta Otolaryngol. Supp. 126, 102 (2006).

[7] H. Schaaf, C. Eichenberg, G. Kastellis, G. Hesse, Otolaryngol. Pol. 64, 78 (2010) (in Polish).

[8] E. Ozimek, Postępy w chirurgii głowy $i$ szyi 1, 5 (2005) (in Polish).

[9] M. Pankowska, D. Hojan-Jezierska, E. Skrodzka, E. Szymiec, L. Kubisz, T. Świdzinski, A. Wicher, Acta Phys. Pol. A 119, 1035 (2011).

[10] P.E. Bijur, W. Silver, E.J. Gallagher, Acad. Emerg. Med. 8, 1153 (2001).

[11] L.J. DeLoach, M.S. Higgis, A.B. Caplan, J.L. Stiff, Anesth. Analg. 86, 102 (1998).

[12] P. Supranowicz, Przegl. Epidemiol. 57, 541 (2003) (in Polish). 